\title{
Amygdala Hyperactivity in MAM Model of Schizophrenia is Normalized by Peripubertal Diazepam Administration
}

\author{
Yijuan Du' and Anthony A Grace ${ }^{*, 1,2,3}$ \\ 'Department of Neuroscience, University of Pittsburgh, Pittsburgh, PA, USA; ${ }^{2}$ Department of Psychiatry, University of Pittsburgh, Pittsburgh, PA, \\ USA; ${ }^{3}$ Department of Psychology, University of Pittsburgh, Pittsburgh, PA, USA
}

\begin{abstract}
In addition to prefrontal cortex (PFC) and hippocampus, amygdala may have a role in the pathophysiology of schizophrenia, given its pivotal role in emotion and extensive connectivity with the PFC and hippocampus. Moreover, abnormal activities of amygdala may be related to the anxiety observed in schizophrenia patients and at-risk adolescents. These at-risk subjects demonstrated heightened levels of anxiety, which are correlated with the onset of psychosis later in life. Similarly, rats that received methyl azoxymethanol acetate (MAM) gestationally exhibited higher levels of anxiety peripubertally. In the current study, the heightened anxiety was also observed in adult MAM animals, as well as higher firing rates of BLA neurons in both peripubertal and adult MAM rats. In addition, the power of BLA theta oscillations of adult MAM rats showed a larger increase in response to conditioned stimuli (CS). We showed previously that administration of the antianxiety drug diazepam during the peripubertal period prevents the hyperdopaminergic state in adult MAM rats. In this study, we found that peripubertal diazepam treatment reduced heightened anxiety, decreased BLA neuron firing rates and attenuated the CS-induced increase in BLA theta power in adult MAM rats, supporting a persistent normalization by this treatment. This study provides a link between BLA hyperactivity and anxiety in schizophrenia model rats and that circumvention of stress may prevent the emergence of pathology in the adult.

Neuropsychopharmacology (2016) 4I, 2455-2462; doi:I0.1038/npp.2016.42; published online 13 April 2016
\end{abstract}

\section{INTRODUCTION}

Schizophrenia is a debilitating mental disorder that involves abnormalities across multiple brain regions in which the prefrontal cortex (PFC; Volk and Lewis, 2002) and hippocampus (Nelson et al, 1998; Benes, 1999) are the most strongly implicated. Another region, the amygdala, has garnered increasing attention, particularly as it relates to the pathophysiology of schizophrenia. Abnormalities in amygdala structure, molecular profile, and physiological activity (Benes, 2010), as well as disruptions of amygdala response to emotional stimuli (Hall et al, 2008), have been reported in schizophrenia patients. Given its pivotal role in emotional function, alterations in the amygdala may underlie emotional and social deficits reported in schizophrenia. The basolateral amygdala (BLA) is extensively interconnected with the hippocampus and PFC (Sah et al, 2003), with malfunction in these circuits leading to cognitive deficits related to stress and emotion (Kim and Diamond, 2002). Furthermore, pharmacologically disinhibiting the amygdala leads to a decrease in density of hippocampal parvalbumin and GAD65/67 expression (Berretta et al, 2001, 2004),

\footnotetext{
*Correspondence: Dr AA Grace, Department of Neuroscience, University of Pittsburgh, A2 10 Langley Hall, Pittsburgh, PA 15260, USA, Tel: + | 412624 4609, Fax: + 412624 9198, E-mail: Graceaa@pitt.edu Received 9 February 2016; revised 16 March 2016; accepted 17 March 2016; accepted article preview online 22 March 2016
}

analogous to that observed in schizophrenia patients (Volk and Lewis, 2002).

Schizophrenia is widely believed to be a neurodevelopmental disorder, in which alterations start early in development and culminate in the emergence of psychosis usually during late adolescence and early adulthood. Both genetic and environmental factors contribute to the development of schizophrenia. Among those risk factors, stress/sensitivity to stress are thought to contribute to transition into psychosis. Aberrant cortisol levels, stress intolerance, and heightened anxiety levels have been reported in subjects at risk for schizophrenia and are correlated with onset of psychosis later in life (Owens et al, 2005; Yung et al, 2005; Walker et al, 2010; Corcoran et al, 2012; Devylder et al, 2013). By administering a mitotoxin methyl azoxymethanol acetate (MAM) to pregnant dams on gestational day 17 , the offspring tested as adults exhibit behavioral, pharmacological, and anatomical characteristics consistent with schizophrenia (Grace and Moore, 1998; Lodge and Grace, 2009). Heightened anxiety-like behaviors and higher sensitivity to stress were also observed in juvenile and adolescent MAM rats (a blunted yet nonadaptive corticosterone level, more freezing and more ultrasonic vocalizations in response to footshocks (Du and Grace, 2013; Zimmerman et al, 2013).

In this study, we examined both single-unit activities of amygdala neurons and oscillatory activities recorded in the amygdala in control and MAM-treated rats. We found that 
BLA hyperactivity was present in peripubertal MAM rats and persisted to adulthood. Correlated with heightened activity of the BLA, MAM rats showed more anxiety-like behaviors in both peripubertal period and adulthood, as well as greater fear conditioning peripubertally. The administration of the antianxiety drug diazepam during the peripubertal period has been shown to prevent the hyperdopaminergic state in adult MAM rats (Du and Grace, 2013). In this study, we showed that peripubertal diazepam administration also leads to a persistent reduction in anxiety-like behaviors and normalization of BLA hyperactivity when assessed in adult MAM rats.

\section{MATERIALS AND METHODS}

\section{Animals}

All procedures were conducted in accordance with the Guide for the Care and Use of Laboratory Animals by the USPHS and approved by the University of Pittsburgh Institutional Animal Care and Use Committee. Pregnant Sprague-Dawley dams were obtained from Harlan on gestational day (GD) 15 and MAM $(20 \mathrm{mg} / \mathrm{kg}$, i.p., Midwest Research Institute, Kansas City, MO) or saline was administered on GD 17. Litters were weaned on postnatal day 23 (PD23) and housed two or three per cage. Only male offspring were used in this study. Animals that were used in LFP recordings and fear conditioning experiments were housed in a reversed light cycle (lights on at 1900 hours and off at 0700 hours); all other animals were housed in a normal light cycle (lights on at 0700 hours and off at 1900 hours). Detailed information of the number of animals and litters used in each experiment can be found in Supplementary Table S1.

\section{Oral Administration of Diazepam}

Diazepam (2 mg tablets, Watson Laboratories, Corona, CA) was ground to powder and mixed with sweetened condensed milk (Eagle Brand), sucrose powder, and ground mini Nilla Wafers (Kraft Food), as described previously (Du and Grace, 2013). For BLA single-unit recordings from peripubertal rats, rats were fed an acute dose of diazepam $(5 \mathrm{mg} / \mathrm{kg})$ mixture or mixture without diazepam (vehicle) $90 \mathrm{~min}$ before behavioral tests or recordings. For tests on adult rats, diazepam $(5 \mathrm{mg} / \mathrm{kg})$ or vehicle was administered during the peripubertal period with once daily administration on 10 consecutive days (PD31-PD40). Visual observation was made throughout feeding to confirm that rats consumed the full dose of diazepam. Rats that had not consumed the entire mixture were excluded from further study. Rats remained drug free after this 10-day administration and were used for recordings or behavioral tests after they reached adulthood (ie, after PD70). The oral administration route was chosen because it is less stressful and it better mimics the preferred route of drug administration to patients (Ferguson and Boctor, 2009).

\section{Acute Surgeries and In Vivo Extracellular Recordings from BLA Neurons in Anesthetized Rats}

Rats were anesthetized with chloral hydrate $(400 \mathrm{mg} / \mathrm{kg}$, i.p.) and supplemented periodically to maintain suppression of the hind limb withdrawal reflex. The body temperature was maintained at $37^{\circ} \mathrm{C}$ using a thermostatic feedback-controlled heating pad (Fintronics, New Haven, CT). A burr hole was drilled in the skull overlying the right BLA. Extracellular recording microelectrodes were pulled from $2.0 \mathrm{~mm}$ glass tubing on a Narishige P-5 vertical electrode puller, the tip broken back under microscopic control, and filled with $2 \mathrm{M}$ $\mathrm{NaCl}$ containing 2\% Chicago Sky Blue dye. The impedance of the electrodes in situ ranged from 7 to $15 \mathrm{M} \Omega$. The stereotaxic coordinates for the BLA were $3.3 \mathrm{~mm}$ posterior to bregma and $5.0 \mathrm{~mm}$ right from the midline for adults and $2.5 \mathrm{~mm}$ posterior and $4.5 \mathrm{~mm}$ right for peripubertal rats (Sherwood and Timiras, 1970; Paxinos and Watson, 2005). Single-unit activity was collected using a high-pass filter at $30 \mathrm{~Hz}$ and low pass at $10 \mathrm{kHz}$. Spontaneously active neurons (firing rate $>0.1 \mathrm{~Hz}$ ) were searched $6.0-8.5 \mathrm{~mm}$ ventral from brain surface. Once identified, activity was recorded for at least $3 \mathrm{~min}$ using the Labchart software (ADInstrument, Colorado Springs, CO).

\section{Elevated Plus Maze}

The elevated plus maze was positioned $50 \mathrm{~cm}$ above the floor. It had four arms, each $50 \mathrm{~cm}$ long and $10 \mathrm{~cm}$ wide, arranged in a cross-like pattern, with two opposite arms enclosed by $40 \mathrm{~cm}$ high opaque walls, the other two without any walls, and a central platform at their intersection $\left(10 \times 10 \mathrm{~cm}^{2}\right)$. Rats were handled for 3 days and habituated to the dark testing room the day before the test. During the test, each rat was placed on the central platform facing an open arm, and its movement was recorded for $5 \mathrm{~min}$. The proportion of time spent in the open arms and the percentage of entries into the open arms were calculated as indices of anxiety-like behaviors.

\section{Survival Surgeries}

Survival surgical procedures were performed in a semisterile environment. Adult (250-350 g) and peripubertal rats (PD28-PD31, 120-150 g) were anesthetized with isoflurane ( $5 \%$ in oxygen for induction, around $2 \%$ for maintenance) and placed in a stereotaxic apparatus using blunt ear bars. Body temperature was maintained at $37^{\circ} \mathrm{C}$. Custom length $(40 \mathrm{~mm})$ polyimide-insulated stainless steel wire electrodes (Plastics One: E363/3, $0.15 \mathrm{~mm}$ ) were implanted bilaterally into BLA (posterior $2.9 \mathrm{~mm}$, left or right $5.0 \mathrm{~mm}$, ventral $8.6 \mathrm{~mm}$ from bregma for adults; posterior $2.5 \mathrm{~mm}$, left or right $4.5 \mathrm{~mm}$, ventral $8.3 \mathrm{~mm}$ for peripubertal rats) (Sherwood and Timiras, 1970; Paxinos and Watson, 2005). A screw attached to a Teflon-insulated stainless steel wire (Plastics One, E363/20, $0.56 \mathrm{~mm}$ ) was fixed above lambda as ground. Another 4-6 (for adults) or 2 (for peripubertal rats) anchor screws were attached to the skull. Electrodes and connectors (E363, Plastics One) were secured with dental cement to the anchor screws. Rats were housed individually after surgery, and received carprofen $(5 \mathrm{mg} / \mathrm{kg}$, i.p.) and Tylenol ( $1 \mathrm{ml}$ in $10 \mathrm{~g}$ chow) for 3 days. Behavioral trainings started 7 days after surgery.

\section{Standard Fear Conditioning and Local Field Potential (LFP) Recordings from the BLA}

Behavioral testing was performed during the active period (between 0700 and 1900 hours). Rats were trained in a 
standard auditory fear conditioning paradigm. A glass open chamber (Coulbourn Instruments) was used for recording in which rats were habituated to the chamber for 2 days (Days 1 and 2). On Day 3, rats were exposed in a different operant chamber (MedAssociates) to 10 trials (intertrial interval ranging from 45 to $100 \mathrm{~s}$ ) of $2 \mathrm{~s}$ tone presentations that ended with a $1 \mathrm{~s}$ low amplitude footshock $(0.45 \mathrm{~mA})$. Twenty-four hours after conditioning (Day 4), rat were placed in the same open glass chamber as in Days 1 and 2. LFP electrodes were connected with a 12-channel commutator (Plastics One) to a 4-channel amplifier (A-M system, model 1700). After at least 20 min habituation to the chamber, rats were exposed to 10 trials of $2 \mathrm{~s}$ tones alone and LFP signals in BLA were recorded. LFP signals were amplified 10000 times, filtered (low pass: $1 \mathrm{kHz}$ and high pass: $1 \mathrm{~Hz}$ ), and digitized at $1000 \mathrm{~Hz}$ in LabChart (ADInstrument). Freezing behaviors were defined as immobility that last at least $2 \mathrm{~s}$ and hand scored for $45 \mathrm{~s}$ after tone presentation.

\section{Histology}

For BLA single-unit recordings, electrode sites were marked at the end of recordings via electrophoretic ejection of Chicago Sky Blue dye from the tip of the electrode. Rats were then overdosed with chloral hydrate and decapitated. For LFP recordings from awake, freely moving rats, after recordings, recording sites were marked by passing a 0.1 $\mathrm{mA}$ current for $10 \mathrm{~s}$ through the electrode and rats were killed with carbon dioxide. Brains were removed and fixed in $8 \%$ paraformaldehyde in PBS for $72 \mathrm{~h}$, immersed in $25 \%$ sucrose in PBS, sliced, and stained with Neutral red/Cresyl violet. Recording sites were verified by microscopic localization of the blue dye mark and electrode tracks. Only neurons located in the BLA complex (both BLA and lateral amygdala) were used for analysis.

\section{Data Analysis and Statistics}

In the analysis of anxiety-like behaviors in the elevated plus maze experiment, a total of five rats were excluded due to equipment failure and failure to initiate movements, and two for values that were $>2$ SD from the mean. In the analysis of freezing behaviors, a total of four adult animals were excluded due to equipment failure and one peripubertal animal for values that were $>2$ SD from the mean.

For BLA single-unit activity, only neurons that exhibited action potential durations $>0.7 \mathrm{~ms}$ and firing rates $<1 \mathrm{~Hz}$ were included in order to preferentially record from projection neurons. There are indeed some projection neurons that fire $>1 \mathrm{~Hz}$; however, using these criteria, the population included for analysis should be comprised almost exclusively of projection neurons (Rainnie et al, 1993; Rosenkranz and Grace, 1999; Likhtik et al, 2006). Firing rates were transformed via a logarithmic function to fit a normal distribution. One-way ANOVA with Bonferroni post-hoc tests were performed to compare Sal- and MAMtreated peripubertal rats; for adult rats, two-way ANOVA tests were used to compare effects of prenatal MAM and peripubertal diazepam administration.

Analysis of LFPs was performed using the chronux package (www.chronus.org) in MATLAB (MathWorks). In brief, LFP signals were filtered $(3-20 \mathrm{~Hz})$, detrended, and
Fourier transformed into different frequency bands. Continuous multitaper time-frequency spectral analyses were conducted for data from $2 \mathrm{~s}$ before to $4 \mathrm{~s}$ after onset of the tone. LFP signals were normalized to those recorded $2 \mathrm{~s}$ before the onset of the tone. The ratio of theta $(4-8 \mathrm{~Hz})$ power after the onset of the tone $v s$ baseline was calculated by normalizing theta power during the 2-s tone presentation to the period $2 \mathrm{~s}$ before the onset of the tone. Trials with saturated signals were excluded and the remainders within each animal were averaged. Animals with data from less than five trials were excluded from the analysis. There was no significant difference between left and right BLA; we thus pooled data from left and right BLA. For rats with data from bilateral BLA, we only used those recorded from the right side.

All statistical tests were performed using SigmaPlot (SigmaStat Software), Matlab (MathWorks), or SPSS (IBM Software). Data are presented as mean \pm SEM.

\section{RESULTS}

\section{Decreased Time and Entries into Open Arms in the Elevated Plus Maze}

We showed previously that MAM-treated rats had higher levels of anxiety as tested in the elevated plus maze peripubertally (Du and Grace, 2013). We now report that adult MAM rats showed a similar increase in anxiety-like behaviors, as indicated by a decrease in the percentage of time spent in the open arms and entries into open arms. MAM rats with peripubertal diazepam treatment spent a significantly higher proportion of time and entries in open arms compared with MAM:Veh rats and were not significantly different from Sal-treated rats (Figure 1; time in open arms: two-way ANOVA significant effect of MAM $\left(\mathrm{F}_{1,42}=8.006, p<0.01\right)$, diazepam $\left(\mathrm{F}_{1,42}=4.264, p<0.05\right)$, and their interaction $\left(\mathrm{F}_{1,42}=5.806, p<0.05\right)$; entries into open arms: two-way ANOVA significant effect of MAM $\left(\mathrm{F}_{1,42}=4.600, p<0.0105\right)$, and interaction between MAM and diazepam $\left(\mathrm{F}_{1,42}=4.413, p<0.05\right)$. Bonferroni post-hoc tests revealed a significant difference between MAM:Veh and MAM:DZ within MAM groups and a significant difference between MAM:Veh and Sal:Veh within Veh groups but not between Sal:Veh and Sal:DZ or Sal:DZ and MAM:DZ.

\section{Increased Firing Rates of BLA Neurons in both Peripubertal and Adult MAM-Treated Rats}

The BLA is a key component in the fear/anxiety circuit (LeDoux, 2000). Therefore, alterations in anxiety levels of MAM-treated rats should be reflected by alterations in BLA neuron activity. The heightened level of anxiety was observed in MAM rats at both peripubertal and adult ages. We thus conducted in vivo single-unit recordings from the BLA of anesthetized rats at both ages.

Similar to that reported previously (Rosenkranz and Grace, 1999), firing rates of spontaneously active BLA neurons were low $(n=13$ cells $/ 5$ rats, $0.11 \pm 0.02 \mathrm{~Hz}$ in peripubertal control rats, Figure 2a; $n=33$ cells/ 5 rats, $0.21 \pm 0.03 \mathrm{~Hz}$ in adult control rats, Figure 2b). At the peripubertal age of PD35PD42, BLA neurons in MAM rats exhibited significantly higher spontaneous firing rates $(n=30 / 6$ rats, $0.41 \pm 0.04 \mathrm{~Hz})$ 
compared with Sal rats. MAM rats with a single dose of diazepam $(5 \mathrm{mg} / \mathrm{kg}$, oral) had significantly lower BLA neuron firing rates $(n=12 / 4$ rats, $0.14 \pm 0.02 \mathrm{~Hz})$ compared with MAM rats without diazepam (one-way ANOVA among
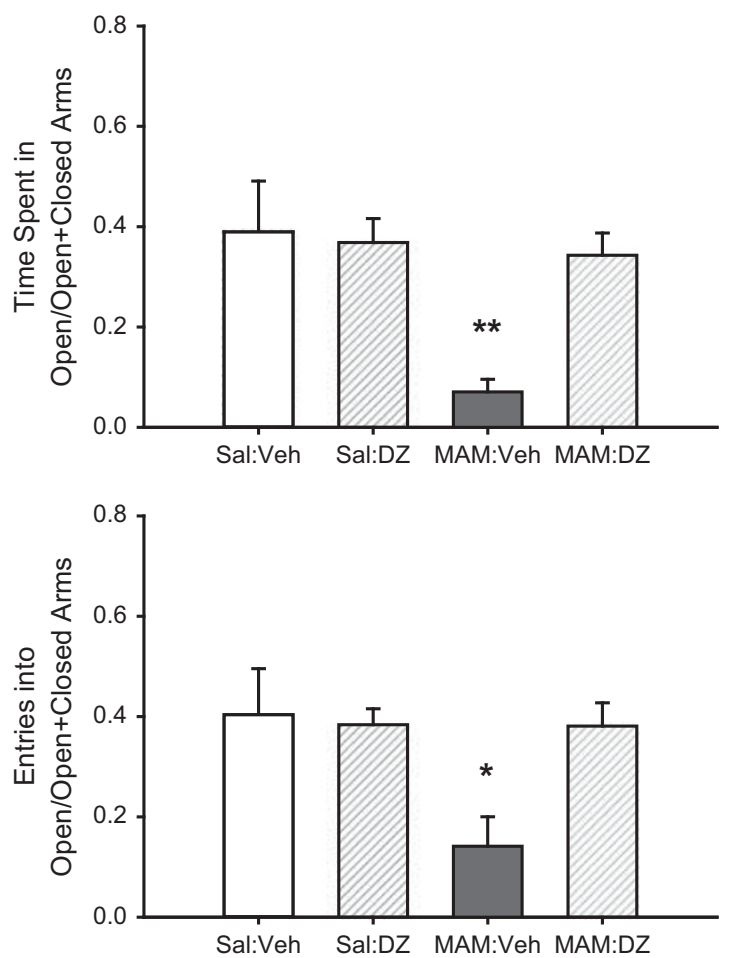

Figure I Peripubertal diazepam administration decreased the heightened level of anxiety in adult MAM rats. MAM rats (MAM:Veh, $n=10$ ) spent a significantly less lower proportion of time in open arms (upper panel) and made significantly less proportion of open arms entries (lower panel) compared with Sal rats (Sal:Veh, $n=10$ ) and Sal rats with peripubertal diazepam administration (Sal:DZ, $n=9$ ), indicative of a higher level of anxiety in MAM rats. In contrast, the anxiety level of MAM rats with peripubertal diazepam administration (MAM:DZ, $n=17$ ) did not differ significantly from that of Sal rats and was significantly lower than MAM:Veh rats. $* p<0.05 ; * * * 0.01$. all three groups revealed a significant effect of treatment $\left(\mathrm{F}_{2,53}=28.425, p<0.001\right)$; Bonferroni post-hoc tests revealed significant difference between MAM and Sal rats $(t=9.471$, $p<0.001$ ), between MAM and MAM with acute diazepam administration $(t=4.914, p<0.001)$, and between Sal and MAM with acute diazepam administration $t=2.642$, $p=0.028)$.

In adults, BLA neurons in MAM:Veh rats exhibited a significantly higher spontaneously firing rate $(n=39 / 6$ rats, $0.41 \pm 0.04 \mathrm{~Hz})$ compared with Sal:Veh $(t=4.096 p<0.001)$ and MAM:DZ $(n=29 / 6$ rats, $0.28 \pm 0.04 \mathrm{~Hz}, t=2.732$, $p=0.043$; two-way ANOVA among all four groups revealed a significant effect of both MAM $\left(\mathrm{F}_{1,123}=19.778, p<0.001\right)$ and diazepam treatment $\left.\left(\mathrm{F}_{1,123}=7.741, p<0.01\right)\right)$. Neuron activities from Sal:Veh, Sal:DZ $(n=26 / 5$ rats, $0.17 \pm 0.04 \mathrm{~Hz})$, and MAM:DZ rats were not significantly different.

\section{Increased Theta Power during Conditioned Tone Presentation in Adult MAM Rats: Normalization Following Peripubertal Diazepam Administration}

The oscillatory activities in the amygdala, especially those in theta frequency have been related to fear and anxiety (Seidenbecher et al, 2003; Pelletier and Pare, 2004; Likhtik et al, 2014). Therefore, we recorded LFP from the BLA of awake, freely moving rats. LFP recordings were conducted after rats were trained in a standard auditory fear conditioning paradigm, an amygdala-dependent task that measures learned fear. Although fear conditioning behaviors reflect learned fear while anxiety-like behaviors as measured in the elevated plus maze reflects innate fear, the two behaviors are highly related (LeDoux, 2000). In this fear conditioning paradigm, rats were recorded in a different context from where they were conditioned. Therefore, the response we observed should reflect only cue-induced but not contextual fear. We did not observe significant effects of MAM on freezing behaviors $45 \mathrm{~s}$ following onset of conditioned tones (Figure 3a). However, peripubertal administration of diazepam significantly reduced freezing behaviors $\left(n=6-10, \mathrm{~F}_{1,26}=5.45, p=0.028\right)$. In contrast,
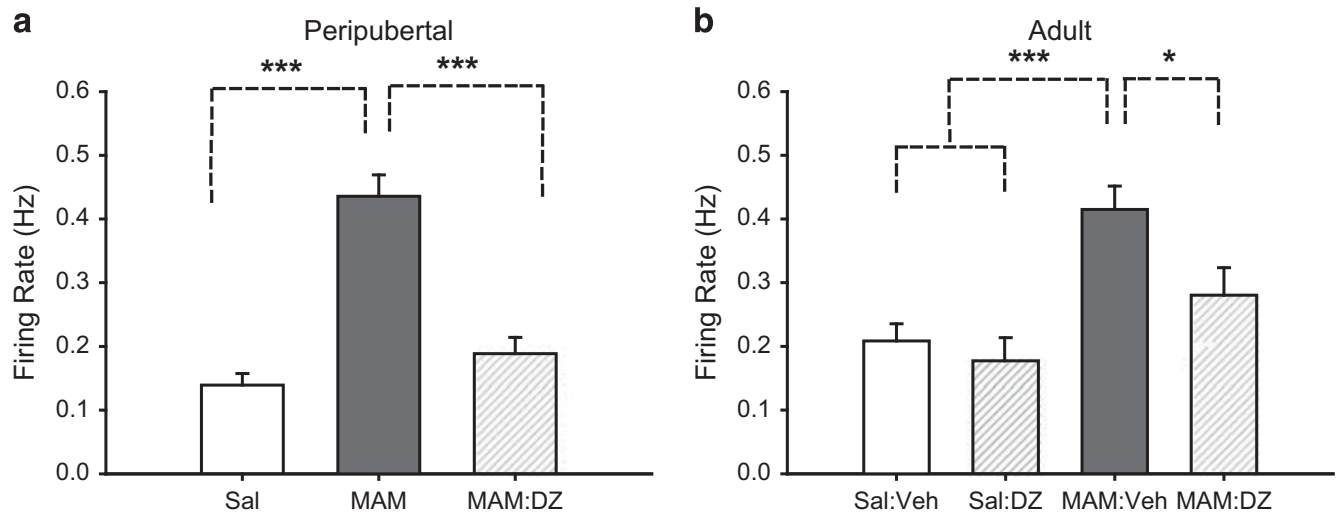

Figure 2 (a) MAM rats exhibited heightened spontaneous activity of BLA neurons peripubertally (PD35-PD42). When measured peripubertally, BLA neurons in MAM rats exhibited significantly $(p<0.00 \mathrm{I})$ higher spontaneous firing rates compared with Sal rats. This BLA hyperactivity in MAM rats was significantly lower $(p<0.00 \mathrm{I})$ in MAM rats with acute administration of diazepam ( $5 \mathrm{mg} / \mathrm{kg}$, oral) before the recording (MAM+acute DZ). (b) Peripubertal administration of diazepam prevented the increase in BLA neuron spontaneous activity in adult MAM rats. BLA neurons in adult MAM rats exhibited significantly $(p<0.00 \mathrm{I}$ ) higher spontaneous firing rates (MAM:Veh), compared with Sal rats (Sal:Veh). In contrast, BLA neuron spontaneous firing rates in MAM rats with peripubertal treatment of diazepam (MAM:DZ) were significantly $(p<0.05)$ lower compared with MAM:Veh. $* p<0.05 ; * * * * 2<0.001$. 
a

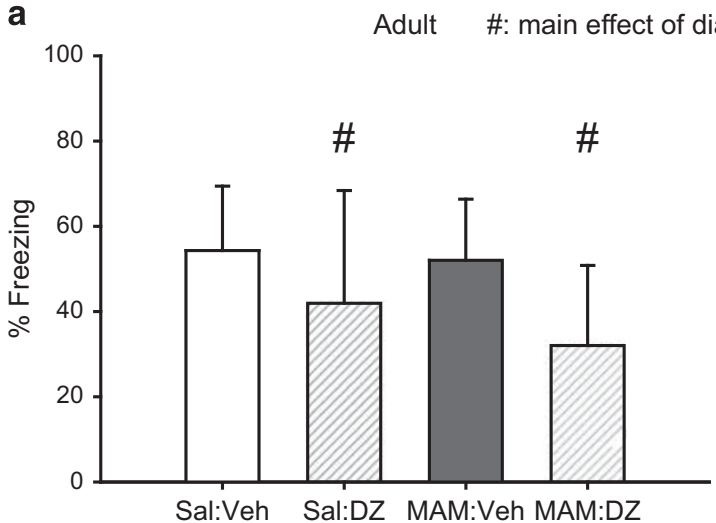

b

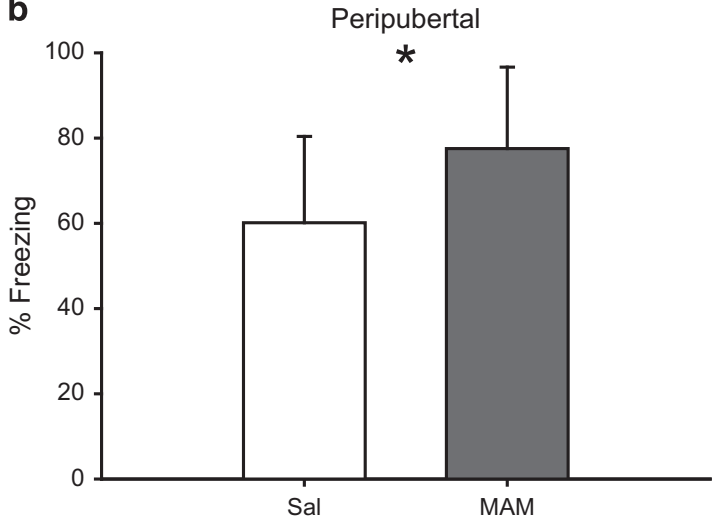

Figure 3 MAM rats showed significantly more freezing behaviors during the peripubertal period but not in adulthood. (a) Although diazepam decreased freezing behaviors in adult MAM and saline rats, adult MAM rats did not exhibit differences in freezing compared with controls, independent of diazepam treatment. (b) In contrast, when measured peripubertally, MAM rats showed significantly more freezing behaviors to conditioned tones. Freezing behaviors were defined as immobility for at least $2 \mathrm{~s}$ and were calculated for $45 \mathrm{~s}$ after the onset of the tone. \#Main effect of diazepam treatment yielded by two-way ANOVA, $p<0.05$; $p<0.05$.

peripubertal MAM rats (PD38-PD42) exhibited significantly more freezing behaviors $(78 \pm 19 \%, n=14)$ compared with Sal rats (Figure $3 b, 60 \pm 20 \%, n=12, t_{24}=-2.254, p=0.034$ ).

LFP signals recorded when these rats were exposed to conditioned tones revealed an increase in theta power during the 2-s period of tone presentation compared with baseline ( $2 \mathrm{~s}$ before the tone presentation) (Figure $4 \mathrm{a}-\mathrm{c}$ ), which is similar to what has been reported previously (Seidenbecher et al, 2003; Likhtik et al, 2014). This increase in theta power during tone presentation is correlated with freezing behaviors following the tone in Sal-treated rats $\left(R^{2}=0.75\right.$, Figure 4d); however, no such correlation was observed in MAM-treated rats $\left(R^{2}=0.0004\right)$. Comparing all four groups of adult rats, no differences in the peak theta power was observed among these animals but the increase in theta power averaged across 2-s tone presentation (Sal:Veh: $n=6$, $141 \pm 10 \%$; Sal:DZ: $n=6,165 \pm 18 \%$; MAM:Veh: $n=5,225 \pm$ 31\%; MAM:DZ: $n=5158 \pm 7 \%$, Figure $4 \mathrm{e}$ and $\mathrm{f}$ ) showed significant effects of MAM $\left(\mathrm{F}_{1,18}=4.42, p=0.0499\right)$ and the interaction of MAM and diazepam treatment $\left(\mathrm{F}_{1,18}=6.03\right.$, $p=0.024$ ) by two-way ANOVA. Benforroni post-hoc tests revealed a significant effect of diazepam within the MAM group (MAM:Veh $v s$ MAM:DZ, $t=2.243, p=0.025$ ) and a significant effect of MAM $v s$ the vehicle group (MAM:Veh $v s$ Sal:Veh, $t=3.222, p=0.005$ ).

Changes in theta power during tone presentation did not differ significantly between peripubertal MAM and saline rats (peripubertal Sal: $124 \pm 28 \%, n=7$; MAM: $99 \pm 14 \%$, $n=7$ ) and was significantly less than in MAM adults. Twoway ANOVA and post-hoc tests revealed a significant interaction between age and MAM treatment $\left(\mathrm{F}_{1,21}=13.6\right.$, $p=0.001)$ and of age $\left(\mathrm{F}_{1,21}=2.12, p<0.001\right)$, and post-hoc tests revealed a significant difference in MAM rats between peripubertal and adult ages $(t=5.865, p<0.001)$.

\section{DISCUSSION}

We found significantly higher anxiety-like behaviors in adult MAM rats as tested in the elevated plus maze, similar to that observed in rats tested peripubertally ( $\mathrm{Du}$ and Grace, 2013). Anxiety is prevalent in schizophrenia patients, with a comorbidity rate of $38.3 \%$ (Achim et al, 2011). The heightened level of anxiety observed in our MAM model of schizophrenia is consistent with this high comorbidity.

Altered function in the amygdala has been advanced as an underlying condition in both anxiety and schizophrenia (LeDoux, 2000; Benes, 2010). Increased anxiety and fear conditioning behaviors are related to BLA hyperactivity. This includes both single-unit and oscillatory activity (Seidenbecher et al, 2003; Pelletier and Pare, 2004; Likhtik et al, 2014). Although not a prominent component under baseline conditions, theta oscillations in the BLA become strong in response to conditioned stimulis and are related with freezing behaviors (Paré and Collins, 2000; Seidenbecher et al, 2003). In this study, neurons in the BLA of MAM rats at both peripubertal and adult ages exhibited significantly higher spontaneously firing rates. In addition, adult MAM rats showed significantly larger increases in theta power in response to conditioned tones as tested in a standard fear conditioning paradigm. The relationship between increases in BLA theta power and freezing behaviors in response to conditioned tone was supported by correlation observed in Sal-treated rats. It is noted that this correlation was examined in a small sample size $(n=5)$. Therefore, such a correlation needs to be tested further using a larger sample. We did observe a significantly larger increase in theta power in adult MAM rats; however, this increase was not reflected in increased freezing behaviors of these rats. The discrepancy in theta power increase and lack of change in freezing behaviors of MAM rats could reflect the contribution of other brain regions to learning or expression of fear. Indeed, both ventral hippocampus and the PFC that are important in fear learning (Rosenkranz and Grace, 2002; Maren and Quirk, 2004) exhibited abnormal function in MAM-treated rats (Goto and Grace, 2006; Lodge and Grace, 2007; Lodge et al, 2009). Synchronized activities of the amygdala with the hippocampus and the PFC in theta band frequencies have been correlated with fear and anxiety behaviors (Seidenbecher et al, 2003; Likhtik et al, 2014; Harris and Gordon, 2015). Therefore, it is possible that, although theta power in BLA is larger in MAM rats, the synchrony between BLA and these brain regions was disrupted, which contributes to the lack of correspondence between freezing behaviors and BLA theta power in these rats. 
Interestingly, the alterations in BLA neuron activities in MAM rats started as early as the peripubertal period and persisted into adulthood, which is consistent with the heightened level of anxiety observed in both peripubertal (Du and Grace, 2013) and adult rats. Indeed, although the onset of psychosis usually occurs during late adolescence and
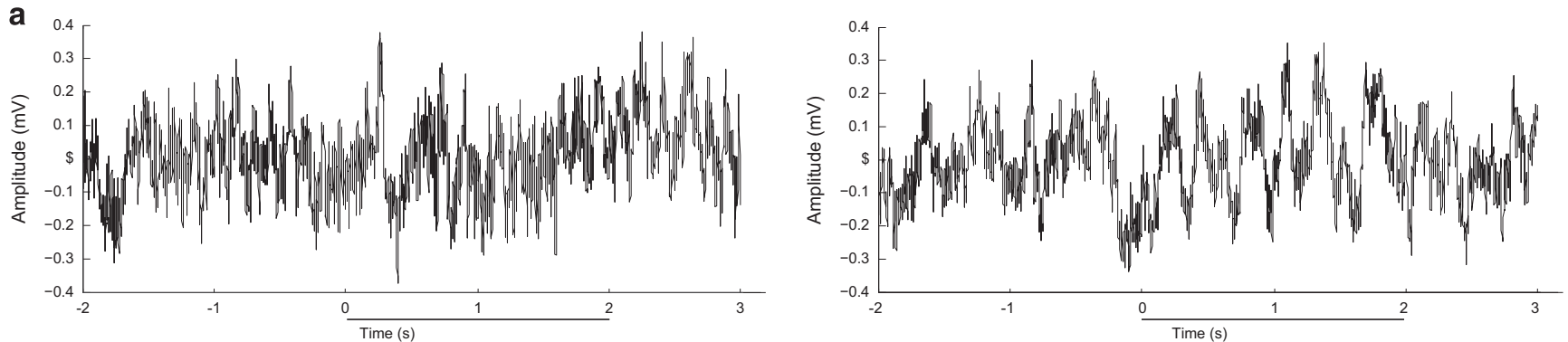

b
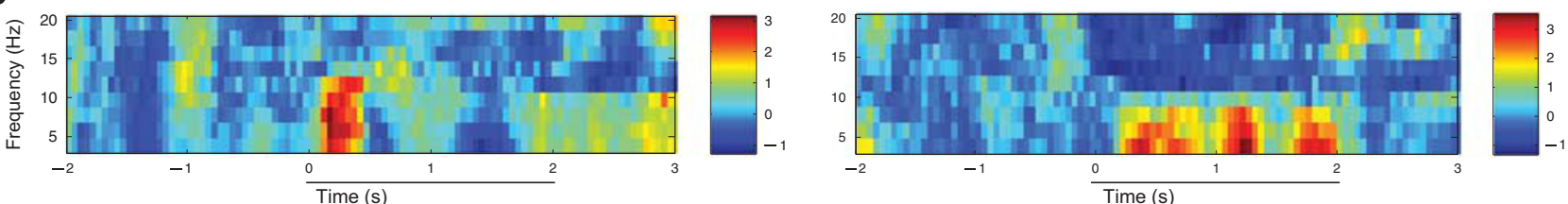

C
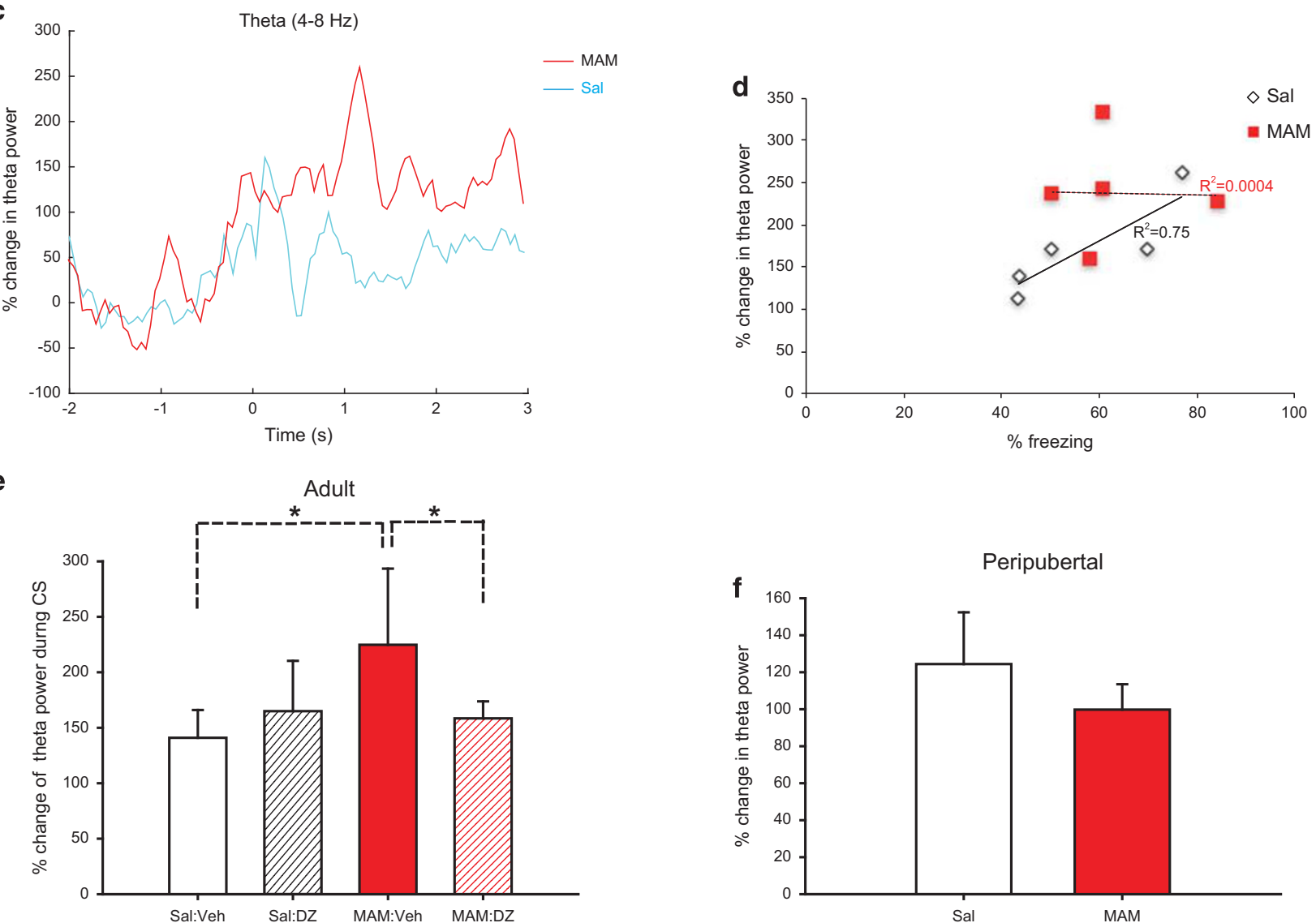

Figure 4 Adult MAM-treated rats exhibited a larger increase in CS-induced theta power than both Sal and MAM rats with peripubertal diazepam treatment. (a) Raw traces of LFP signal examples from one Sal- and one MAM-treated rat. The bar depicts tone presentation from 0 to $2 \mathrm{~s}$. (b) LFP signals were Fourier transformed to different frequency bands, normalized to the baseline ( $2 \mathrm{~s}$ immediately before the onset of the tone) and average across trials for each animal. The power spectrograms from example animals are represented as z-scores in heat maps for Sal- and MAM-treated rats. (c) The average power spectrogram of theta frequency $(4-8 \mathrm{~Hz})$ of Sal- and MAM-treated rats, in which the red line represents MAM rats and the blue represents Sal rats. (d) A correlation between freezing behaviors and changes in CS-induced theta power was observed in Sal:Veh but not in MAM:Veh animals. The trend lines were shown for the Sal:Veh group as a solid line and for MAM:Veh as dashed. $R^{2}$ values were shown for the Sal:Veh group as black and for MAM:Veh as red. (e) The percentage of increase in theta power during the 2-s tone presentation is normalized to baseline (2 s period immediately before the onset of tone) for all the four groups. MAM:Veh animals exhibit a significantly greater increase in BLA theta power compared with the other groups. $(f)$ The percentage of change in theta power during the 2-s tone presentation was not significantly different between peripubertal Sal and MAM rats. * $p<0.05$. 
early adulthood, alterations in behaviors and brain structures initiate earlier. Subjects at risk for schizophrenia demonstrate a variety of prodromal symptoms, including altered emotional and cognitive functions (Johnstone et al, 2005), as well as hypermetabolism in the hippocampus (Schobel et al, 2013). Similarly, in our MAM-treated rats, the hyperlocomotion in response to psychostimulants is only observed in adults but not in younger rats (Moore et al, 2006). However, at juvenile and peripubertal ages, these MAM rats already exhibit a significant degree of pathology: they are more sensitive to stress and more anxious (Du and Grace, 2013; Zimmerman et al, 2013), and the reduction of parvalbumin expression in the hippocampus initiates in juvenile MAM rats (Chen et al, 2014; Gill and Grace, 2014).

We confirmed our previous studies showing the antianxiety effect of diazepam in peripubertal MAM rats (Du and Grace, 2013) and now show a persistent antianxiety effect of peripubertal diazepam in adult MAM rats after remaining drug free for at least a month. Thus those with previous diazepam treatment during the peripubertal period exhibited significantly lower levels of anxiety, lower BLA neuron spontaneously firing rates, and less increase in conditioned tone-induced theta power compared with nontreated MAM rats. This peripubertal period, corresponding to mid-to-late adolescence in humans, is a critical period for prefrontal cortical and inhibitory circuit development, both involved in the pathophysiology of schizophrenia (Volk and Lewis, 2002). The heightened response to stress in peripubertal MAM rats would make these rats more vulnerable to the effect of stress and influence normal development of these structures. For example, parvalbumin interneurons are sensitive to stress (Czeh et al, 2005; Hu et al, 2010) and would likely be affected. And indeed, the loss of parvalbumin interneurons in the hippocampus initiated in juvenile rats and progressed into adulthood (Gill and Grace, 2014). Loss of parvalbumin in the hippocampus is related to anxiety behaviors (Quarta et al, 2015). Therefore, the persistent impact of peripubertal diazepam administration to reduce anxiety, BLA neuron hyperactivity, and the hyperdopaminergic state in adults could occur via protecting parvalbumin interneurons from further stress-induced damages.

Stabilization of brain systems peripubertally using diazepam in the current study, as well as antipsychotic drugs (Piontkewitz et al, 2009, 2010, 2012) or antioxidants (Cabungcal et al, 2014), effectively prevent the emergence of schizophrenia-like symptoms in developmental disruption models of schizophrenia. A common variable appears to be the contribution of stress to the development of schizophrenia. Schizophrenia subjects may not necessarily experience more stressful life events, but through genetic predisposition may instead experience these events as more uncontrollable and intolerable (Thompson et al, 2004; van Winkel et al, 2008). Subjects at risk for schizophrenia exhibited higher levels of anxiety and stress intolerance (Owens et al, 2005; Yung et al, 2005), which is correlated with onset of psychosis later in life. This is consistent with the early-onset increase in anxiety behaviors in MAM rats. The fact that diazepam pretreatment can prevent the emergence of a psychosis-like state in rats suggests that MAM does not cause the disorder but instead facilitates the impact of stress on disrupting this system. Thus, unlike genetic disorders such as Huntington's disease, in which the gene causes the disorder, in schizophrenia genetic risk factors may instead function to heighten the deleterious effects of stress by making the individual more vulnerable to the impact of stressful life events, thereby precipitating the subsequent onset of psychosis.

\section{FUNDING AND DISCLOSURE}

AAG has received funds from Johnson \& Johnson, Lundbeck, Pfizer, GSK, Merck, Takeda, Dainippon Sumitomo, Otsuka, Lilly, Roche, Asubio, Abbott, Autofony, and Janssen. YD declares no conflict of interest.

\section{ACKNOWLEDGMENTS}

This work was funded by NIH MH57440 (to AAG). We thank Niki MacMurdo for technical support.

\section{REFERENCES}

Achim AM, Maziade M, Raymond E, Olivier D, Merette C, Roy MA (2011). How prevalent are anxiety disorders in schizophrenia? A meta-analysis and critical review on a significant association. Schizophr Bull 37: 811-821.

Benes FM (1999). Evidence for altered trisynaptic circuitry in schizophrenic1 hippocampus. Biol Psychiatry 46: 589-599.

Benes FM (2010). Amygdalocortical circuitry in schizophrenia: from circuits to molecules. Neuropsychopharmacology 35: 239-257.

Berretta S, Lange N, Bhattacharyya S, Sebro R, Garces J, Benes FM (2004). Long-term effects of amygdala GABA receptor blockade on specific subpopulations of hippocampal interneurons. Hippocampus 14: 876-894.

Berretta S, Munno DW, Benes FM (2001). Amygdalar activation alters the hippocampal GABA system: 'partial' modelling for postmortem changes in schizophrenia. J Comp Neurol 431: 129-138.

Cabungcal JH, Counotte DS, Lewis EM, Tejeda HA, Piantadosi P, Pollock C et al (2014). Juvenile antioxidant treatment prevents adult deficits in a developmental model of schizophrenia. Neuron 83: 1073-1084.

Chen L, Perez SM, Lodge DJ (2014). An augmented dopamine system function is present prior to puberty in the methylazoxymethanol acetate rodent model of schizophrenia. Dev Neurobiol 74: 907-917.

Corcoran CM, Smith C, McLaughlin D, Auther A, Malaspina D, Cornblatt B (2012). HPA axis function and symptoms in adolescents at clinical high risk for schizophrenia. Schizophr Res 135: $170-174$.

Czeh B, Simon M, van der Hart MGC, Schmelting B, Hesselink MB, Fuchs E (2005). Chronic stress decreases the number of parvalbumin-immunoreactive interneurons in the hippocampus: prevention by treatment with a substance $\mathrm{P}$ receptor (NK1) antagonist. Neuropsychopharmacology 30: 67-79.

Devylder JE, Ben-David S, Schobel SA, Kimhy D, Malaspina D, Corcoran CM (2013). Temporal association of stress sensitivity and symptoms in individuals at clinical high risk for psychosis. Psychol Med 43: 259-268.

Du Y, Grace AA (2013). Peripubertal diazepam administration prevents the emergence of dopamine system hyperresponsivity in the MAM developmental disruption model of schizophrenia. Neuropsychopharmacology 38: 1881-1888.

Ferguson SA, Boctor SY (2009). Use of food wafers for multiple daily oral treatments in young rats. J Am Assoc Lab Anim Sci 96: 292-296. 
Gill KM, Grace AA (2014). Corresponding decrease in neuronal markers signals progressive parvalbumin neuron loss in MAM schizophrenia model. Int J Neuropsychopharmacol 17: 1609-1619.

Goto Y, Grace AA (2006). Alterations in medial prefrontal cortical activity and plasticity in rats with disruption of cortical development. Biol Psychiatry 60: 1259-1267.

Grace AA, Moore H (1998). Regulation of information flow in the nucleus accumbens: a model for the pathophysiology of schizophrenia. In: Lenzenweger MF, Dworkin RH (eds). Origins and Development of Schizophrenia: Advances in Experimental Psychopathology. American Psychological Association Press: Washington, DC, USA, pp 123-157.

Hall J, Whalley HC, McKirdy JW, Romaniuk L, McGonigle D, McIntosh AM et al (2008). Overactivation of fear systems to neutral faces in schizophrenia. Biol Psychiatry 64: 70-73.

Harris AZ, Gordon JA (2015). Long-range neural synchrony in behavior. Annu Rev Neurosci 38: 171-194.

Hu W, Zhang M, Czeh B, Flugge G, Zhang W (2010). Stress impairs GABAergic network function in the hippocampus by activating nongenomic glucocorticoid receptors and affecting the integrity of the parvalbumin-expressing neuronal network. Neuropsychopharmacology 35: 1693-1707.

Johnstone EC, Ebmeier KP, Miller P, Owens DGC, Lawrie SM (2005). Predicting schizophrenia: findings from the Edinburgh High-Risk Study. Br J Psychiatry 186: 18-25.

Kim JJ, Diamond DM (2002). The stressed hippocampus, synaptic plasticity and lost memories. Nat Rev Neurosci 3: 453-462.

LeDoux J (2000). Emotion circuits in the brain. Annu Rev Neurosci 23: $155-184$

Likhtik E, Pelletier JG, Popescu AT, Pare D (2006). Identification of basolateral amygdala projection cells and interneurons using extracellular recordings. J Neurophysiol 96: 3257-3265.

Likhtik E, Stujenske JM, Topiwala MA, Harris AZ, Gordon JA (2014). Prefrontal entrainment of amygdala activity signals safety in learned fear and innate anxiety. Nat Neurosci 17: 106-113.

Lodge DJ, Behrens MM, Grace AA (2009). A loss of parvalbumincontaining interneurons is associated with diminished oscillatory activity in an animal model of schizophrenia. J Neurosci 29: 2344-2354.

Lodge DJ, Grace AA (2007). Aberrant hippocampal activity underlies the dopamine dysregulation in an animal model of schizophrenia. J Neurosci 27: 11424-11430.

Lodge DJ, Grace AA (2009). Gestational methylazoxymethanol acetate administration: a developmental disruption model of schizophrenia. Behav Brain Res 204: 306-312.

Maren S, Quirk GJ (2004). Neuronal signalling of fear memory. Nat Rev Neurosci 5: 844-852.

Moore H, Jentsch JD, Ghajarnia M, Geyer MA, Grace AA (2006). A neurobehavioral systems analysis of adult rats exposed to methylazoxymethanol acetate on E17: implications for the neuropathology of schizophrenia. Biol Psychiatry 60: 253-264.

Nelson MD, Saykin AJ, Flashman LA, Riordan HJ (1998). Hippocampal volume reduction in schizophrenia as assessed by magnetic resonance imaging. Arch Gen Psychiatry 33-40.

Owens DGC, Miller P, Lawrie SM, Johnstone EC (2005). Pathogenesis of schizophrenia: a psychopathological perspective. Br J Psychiatry 186: 386-393.

Paré D, Collins DR (2000). Neuronal correlates of fear in the lateral amygdala: multiple extracellular recordings in conscious cats. J Neurosci 20: 2701-2710.

Paxinos G, Watson C (2005). The Rat Brain in Stereotaxic Coordinates, 5th edn. Elsevier Academic Press: San Diego, CA, USA.

Pelletier JG, Pare D (2004). Role of amygdala oscillations in the consolidation of emotional memories. Biol Psychiatry 55: 559-562.
Piontkewitz Y, Arad M, Weiner I (2010). Risperidone administered during asymptomatic period of adolescence prevents the emergence of brain structural pathology and behavioral abnormalities in an animal model of schizophrenia. Schizophr Bull 37: $1257-1269$.

Piontkewitz Y, Assaf Y, Weiner I (2009). Clozapine administration in adolescence prevents postpubertal emergence of brain structural pathology in an animal model of schizophrenia. Biol Psychiatry 66: 1038-1046.

Piontkewitz Y, Bernstein HG, Dobrowolny H, Bogerts B, Weiner I, Keilhoff G (2012). Effects of risperidone treatment in adolescence on hippocampal neurogenesis, parvalbumin expression, and vascularization following prenatal immune activation in rats. Brain Behav Immun 26: 353-363.

Quarta E, Bravi R, Scambi I, Mariotti R, Minciacchi D (2015). Increased anxiety-like behavior and selective learning impairments are concomitant to loss of hippocampal interneurons in the presymptomatic SOD1(G93A) ALS mouse model. J Comp Neurol 523: $1622-1638$.

Rainnie DG, Asprodini EK, Shinnick-Gallagher P (1993). Intracellular recordings from morphologically identified neurons of the basolateral amygdala. J Neurophysiol 69: 1350-1362.

Rosenkranz JA, Grace AA (1999). Modulation of basolateral amygdala neuronal firing and afferent drive by dopamine receptor activation in vivo. J Neurosci 19: 11027-11039.

Rosenkranz JA, Grace AA (2002). Cellular mechanisms of infralimbic and prelimbic prefrontal cortical inhibition and dopaminergic modulation of basolateral amygdala neurons in vivo. $J$ Neurosci 22: 324-337.

Sah P, Faber ESL, Lopez De Armentia M, Power J (2003). The amygdaloid complex: anatomy and physiology. Physiol Rev 83: 803-834.

Schobel SA, Chaudhury NH, Khan UA, Paniagua B, Styner MA, Asllani I et al (2013). Imaging patients with psychosis and a mouse model establishes a spreading pattern of hippocampal dysfunction and implicates glutamate as a driver. Neuron 78: 81-93.

Seidenbecher T, Laxmi TR, Stork O, Pape HC (2003). Amygdalar and hippocampal theta ryhthm synchronization during fear memory retrieval. Science 301: 846-850.

Sherwood NM, Timiras PS. A Stereotaxic Atlas of the Developing Rat Brain. University of California Press: Berkeley, CA, 1970.

Thompson JL, Pogue-Geile MF, Grace AA (2004). Developmental pathology, dopamine, and stress: a model of the age of onset of schizophrenia symptoms. Schizophr Bull 30: 875-880.

van Winkel R, Stefanis NC, Myin-Germeys I (2008). Psychosocial stress and psychosis. A review of the neurobiological mechanisms and the evidence for gene-stress interaction. Schizophr Bull 34: 1095-1105.

Volk DW, Lewis DA (2002). Impaired prefrontal inhibition in schizophrenia: relevance for cognitive dysfunction. Physiol Behav 77: 501-505.

Walker EF, Brennan PA, Esterberg M, Brasfield J, Pearce B, Compton MT (2010). Longitudinal changes in cortisol secretion and conversion to psychosis in at-risk youth. J Abnorm Psychol 119: 401-408.

Yung AR, Yuen HP, McGorry PD, Phillips LJ, Kelly D, Dell'olio M et al (2005). Mapping the onset of psychosis: the comprehensive assessment of at-risk mental states. Aust $N$ Z J Psychiatry 39: 964-971.

Zimmerman EC, Bellaire M, Ewing SG, Grace AA (2013). Abnormal stress responsivity in a rodent developmental disruption model of schizophrenia. Neuropsychopharmacology 38: 2131-2139.

Supplementary Information accompanies the paper on the Neuropsychopharmacology website (http://www.nature.com/npp) 